\title{
ACUMULAÇÃO DIFERENCIAL DE NUTRIENTES POR CINCO CULTIVARES DE MILHO (Zea mays L.). II - ACUMULAÇÃO DE MICRONUTRIENTES*
}

\author{
A.G. DE ANDRADE** \\ H.P. HAAG *** \\ G.D. DE OLIVEIRA *** \\ J.R. SARRUGE***
}

\begin{abstract}
RESUMO
No presente trabalho, os autores apresentam os resultados de um ensaio de campo empregando os cultivares Agroceres 256, Agroceres 504, Centralmex, H-7974 e Piranão no sentido de aquilatar diferenças na acumulação e exportação de micronutrientes. $O$ ensaio foi conduzido num regossol de fertilidade mediana, exceto em relação ao $\mathrm{K}$ que é baixo, situado no município de Piracicaba, SP. O delineamento experimental utilizado foi de blocos ao acaso com 4 repetições. Foram seguidas as práticas culturais comuns e a adubação constituiu de $83 \mathrm{~g}$ da fórmula $30-$ $-120-70$ por metro linear por ocasião do plantio e $33 \mathrm{~g}$ por metro linear da fórmula 50-0-4, em cobertura 22 dias após a germinação. Plantas foram coletadas a partir dos 20 dias após a germinação, em intervalos de 20 dias até os 120 dias. As plantas foram divididas em "colmo + folhas", pendão e espiga e analisadas para $\mathrm{B}, \mathrm{Cu}, \mathrm{Fe}, \mathrm{Mn}$ e $\mathrm{Zn}$. Concluíram os autores que diferenças na acumulação de micronutrientes manifestam-se antes da fase de crescimento intenso. Os cultivares atingem o máximo da quantidade dos nutrientes nas seguintes épocas, em dias: $\mathrm{Cu}(61-85) ; \mathrm{Fe}(71-76) ; \mathrm{Mn}(82-94) ; \mathrm{Zn}(87-108)$. Verifica ram, ainda, que as quantidades máximas extraídas em mg/planta são: $\mathrm{Cu}(2,06-$ $-3,49)$; Fe $(26,66-36,28)$; Mn (9,92-14,39); Zn (5,88-6,69). Finalmente a exportação de nutrientes nas espigas por hectare (50.000 plantas) colhidas é: $\mathrm{Cu}(26-35 \mathrm{~g}) ; \mathrm{Fe}(200-220 \mathrm{~g}) ; \mathrm{Mn}(90-140 \mathrm{~g})$ e $\mathrm{Zn}$ $(160-250 \mathrm{~g})$.
\end{abstract}

\section{INTRODUÇÃO}

A produção final de uma cultura é o reflexo da interação entre diversos fatores que, fundamentalmente, estão ligados ao germoplasma e ao meio ambiente. A produção de $u$ ma planta traduz o potencial de cada um desses fatores e sua integração no individuo.

No passado, os geneticistas procuravam aumentar a produção final, sem se preocupar com o complexo de fatores envolvidos na interação genótipo-ambiente. Contudo, a adaptação da planta ao solo, clima, a resistência às doenças e ao acamamento, constituem hoje uma importante parcela dos estudos de melhoramento. A criação de plantas

* Parte da dissertação apresentada pelo primeiro autor para obtenção do grau de MESTRE pela E.S.A. "Luiz de Queiroz", USP. Suporte financeiro da EMBRAPA, Brasília-DF. Entregue para publicação em 22/07/1975.

* Universidade Federal Rural de Pernambuco, Recife-PE.

*** E.S.A. "Luiz de Queiroz", USP, Depto. de Química, Piracicaba-SP. 
cada vez mais eficientes do ponto de vista fisiológico, é uma das preocupações da genética fisiológica, e que grande contribuições trará para o aumento da produtividade (DUNCAN, 1967; SPRAGUE, 1969; DONALS, 1968).

Muitos processos já se fizeram notar acerca dos aspectos genéticos da absorção, translocação e utilização dos nutrientes minerais (EPSTEIN, 1972).

Os problemas relacionados com a nutrição da planta geralmente se tem tentado resolver modificando as condições de solo. Contudo, há possibilidade de se tirar partido das diferenças genéticas entre plantas, com vistas a adequá-la ao ambiente, com provảveis ganhos na produção, sem alterações muitas vezes dispendiosas nas práticas de cultivo. Este tipo de trabalho já havia sido sugerido, desde 1928, por GREGORY \& CROWTHER (1928).

No Brasil, embora o trabalho de melhoramento tenha alcançado um desenvolvimento elevado, de tal forma que os cultivares apresentam produções de até $15 \mathrm{t} / \mathrm{ha}$ em condições experimentais; não se tem um nivel de conhecimento da fisiologia e nutrição mineral dos cultivares obtidos, compativel com a necessidade, embora inúmeros ensaios de adubação tenham sido conduzidos.

0 presente trabalho tem a finalidade de aquilatar diferenças na acumulação dos micronutrientes e outros parâmetros vinculados, entre os cultivares: Agroceres 256, Agroceres 504, Centralmex, H-7974 e Piranão, com base nas seguintes características: a) acumulação de nutrientes ( $\mathrm{Cu}, \mathrm{Fe}, \mathrm{Mn} \in \mathrm{Zn})$ em função do estádio de desenvolvimento; b) exportação de nutrientes.

\section{MATERIAL E MÉTODOS}

Foram utilizados os seguintes cultivares de milho (Zea mays L.): Agroceres 256, Agroceres 504, Centralmex, H-7974 e Piranão. O experimento foi conduzido no município de Piracicaba, num solo classificado como Regossol, série Sertãozinho (RANNI, 1956), de média fertilidade, exceto em relação ao teor de K que é baixo.

Foram seguidas as práticas culturais comuns e a adubação consistiu de $83 \mathrm{~g}$ da fórmula 30-120-70, por metro linear no plantio, e $33 \mathrm{~g} /$ metro linear da fórmula 50-0-40, em cobertura, 22 dias após germinação.

A população de plantas foi de 50.000 por hectare.

Plantas foram coletadas a partir dos 20 dias após a germinação, em intervalos de 20 dias, até aos 120 dias. As plantas colhidas foram divididas em "colmo + folhas", pendão e espiga, e analisadas quimicamente para $\mathrm{B}, \mathrm{Cu}, \mathrm{Fe}, \mathrm{Mn}$ e $\mathrm{Zn}$ seguidos os métodos descritos em SARRUGE \& HAAG (1974). 


\section{RESULTADOS E DISCUSSÃO}

\section{Acumulação de micronutrientes}

Os resultados obtidos, relativos ao teor e quantidade de micronutrientes nos cinco cultivares estão contidos nos Quadros 1 a 4.

A quantidade dos micronutrientes necessária para promover um crescimento adequado é muito pequena, e a faixa de teores considerada adequada é muito ampla (JONES, 1972; LOCKMAN, 1969; NEUBERT et al., 1969; LUTZ et al., 1972). A variação destes teores com os fatores ambientais, tais como, $\mathrm{pH}$ do solo e presença de outros ions, é elevada (WARNOCK, 1970; LUTZ et al., 1972; OLSEN, 1972); e a ocorrência de falhas na amostragem, preparação e análise quimica, são fatores que contribuem para tornar difícil a generalização sobre o comportamento dos cultivares.

Nos cultivares estudados verificou-se que as diferenças tenderam a aparecer logo no início do ciclo, se bem que estas diferenças fossem eliminadas posteriormente. Vale salientar, que nos estudos da influência do genótipo sobre a absorção de micronutrientes, as análises são feitas aos 30 e 40 dias após a semeadura (veja-se BROWN, 1967;BROWN \& AMBLER, 1970; CLARK \& BROWN, 1974a) quando já se pode notar sintomas de deficiência porventura existentes.

Verificou-se contudo, que as diferenças mais acentuadas entre cultivares apareceram quando a acumulação se aproximou do ponto máximo.

As diferenças de acumulação de micronutrientes encontradas, refletem a interação entre o cultivar e o ambiente, mais que diferenças absolutas na capacidade de extração.

Para se testar diferenças genéticas na capacidade de acumulação de micronutrientes, recomenda-se cultivar plantas em solos deficientes no nutriente considerado, para que tenha contrastes significativos (CLARK \& BROWN, 1974a), pois nos solos com teores normais de micronutrientes, os cultivares tendem a se comportar de forma idêntica (BROWN, 1967; BROWN \& AMBLER, 1970; BROWN \& CLARK, 1974).

Em termos gerais, os cultivares apresentaram algumas diferenças em relação à acumulação de micronutrientes, embora estas não chegassem a merecer destaque especial. As quantidades e teores encontrados estão dentro dos limites citados na literatura.

O B foi analisado apenas na coleta procedida aos 80 dias após a germinação, devido às dificuldades para realizar a análise em todas as amostras. Foi escolhida esta idade por ser próxima do ponto de máximo para quase todos os nutrientes, e porque foi a época e parte da planta em que constatou as diferenças mais expressivas.

Os resultados obtidos acham-se expostos a seguir, e pelo valor de $\mathrm{F}$ nota-je que não houve diferenças entre cultivares. 
Quantidade e teor de B na parte vegetativa dos cultivares aos 80 dias após a germinação.

\begin{tabular}{lcc}
\hline Cultivar & Teor (ppm) & Quantidade (mg/planta) \\
\hline \hline Agroceres 256 & 9,8 & 1,92 \\
Agroceres 504 & 8,7 & 1,73 \\
Centralmex & 7,9 & 1,87 \\
H-7974 & 9,0 & 1,95 \\
Piranão & 10,1 & 1,75 \\
\hline F & & 0,17 \\
D.M.S. (Tukey) 5\% & & n.s. \\
C.V.\% & & 25,2 \\
\hline
\end{tabular}

\section{Cobre}

Os dados analíticos referentes à concentração e quantidade de $\mathrm{Cu}$ nos órgãos dos cultivares acham-se no Quadro 1.

As diferenças encontradas entre cultivares, indicadas pela significância do valor $F$, foram somente para a parte vegetativa aos $20,60,80$ e 100 dias, sendo que aos 80 dias esta diferença não teve tendência a acarretar diferenças na quantidade de $\mathrm{Cu}$ na planta inteira. Aos 120 dias, embora as partes não tenham apresentado diferenças significativas, a quantidade total o foi.

As curvas e equações obtidas através da análise de regressão e que estão representadas na Figura 1, visualizam as diferenças entre cultivares em relação à quantidade total de $\mathrm{Cu}$ na planta. 


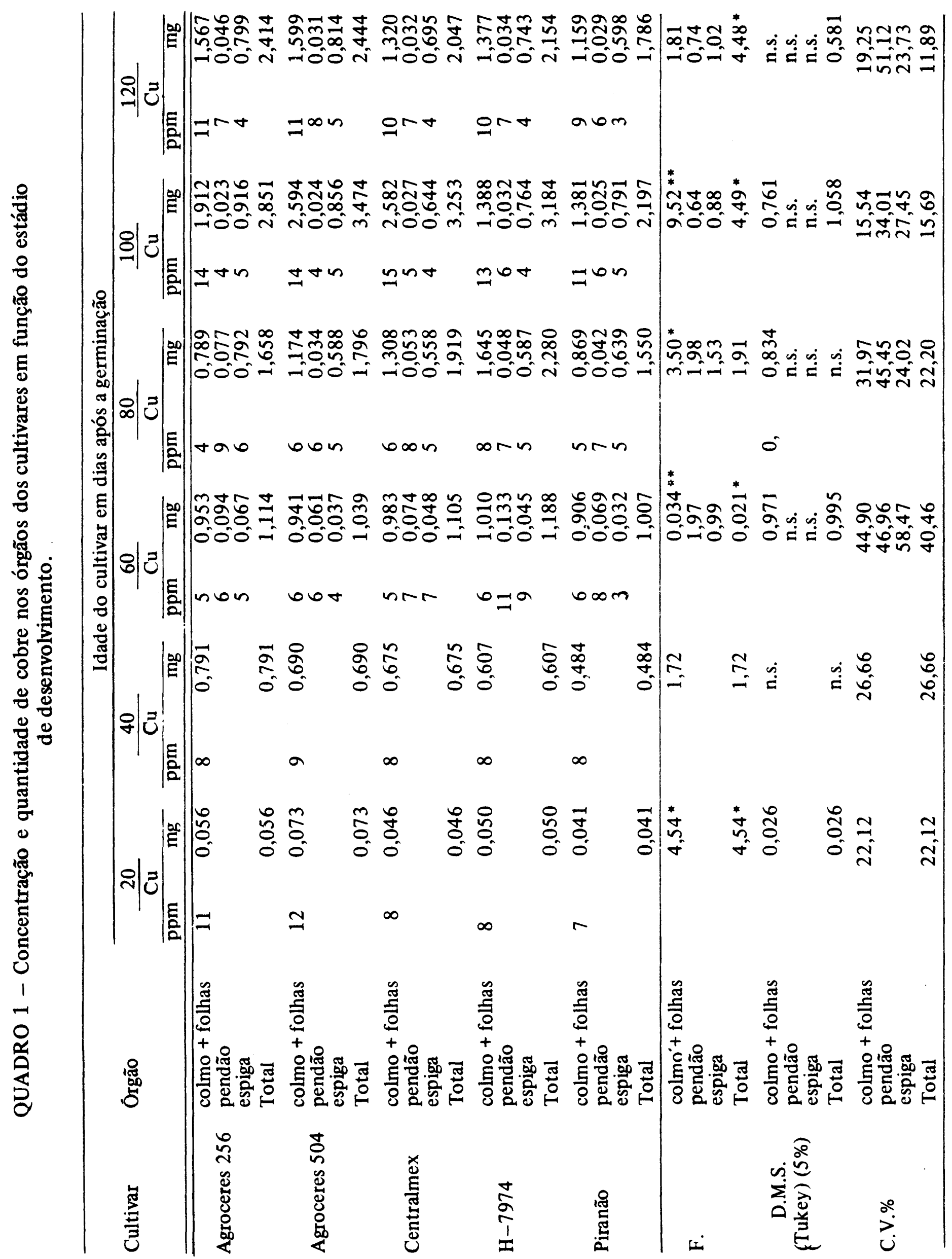




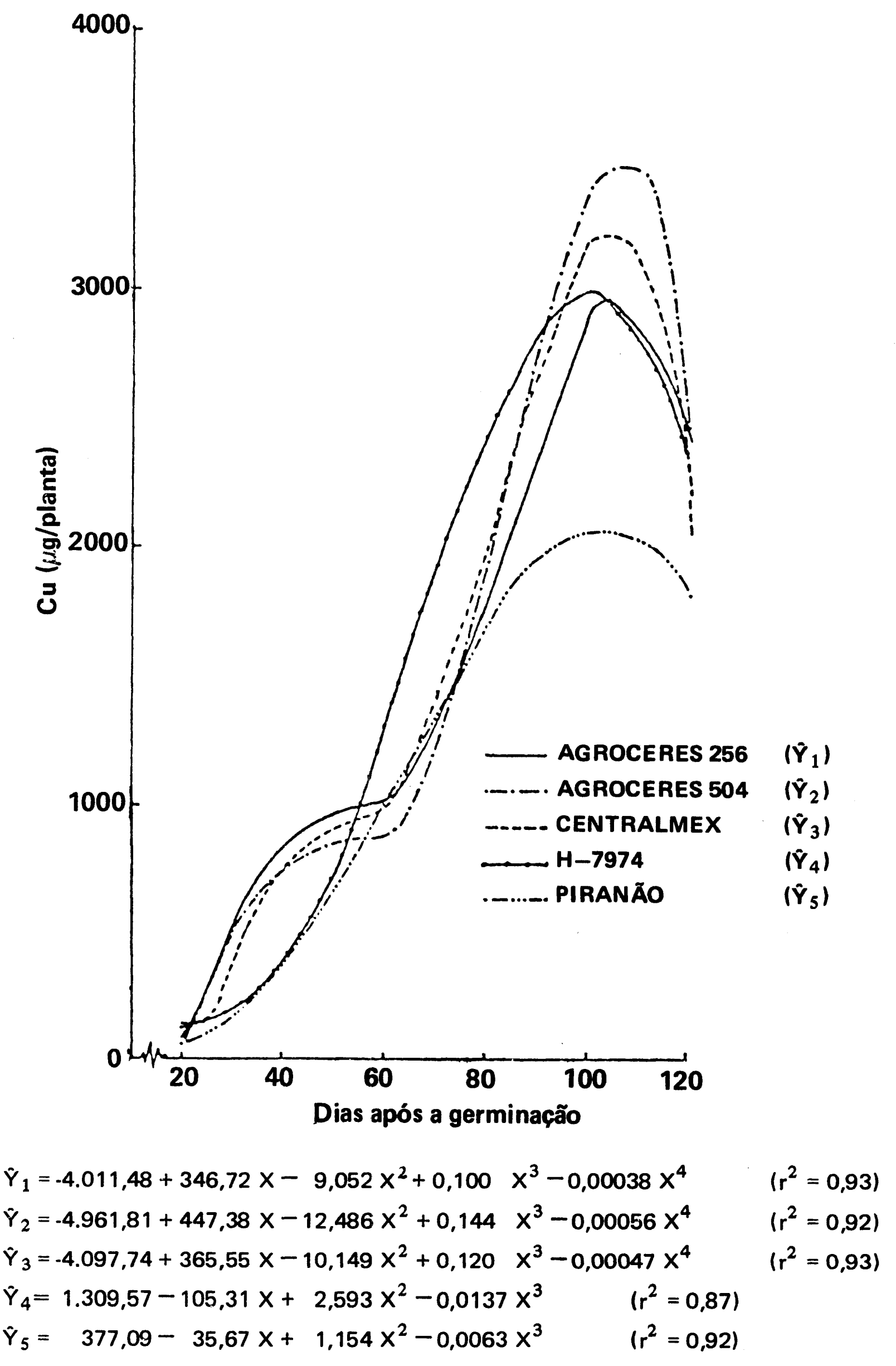

Figura 1 - Curvas de regressão da quantidade de cobre $(\hat{\mathbf{Y}})$ em função da idade (X) nos culțivares. 
Os cultivares Agroceres 256 e 504, e o Centralmex, seguiram curvas do 4 9 grau devido a uma diminuição relativa na taxa de acumulação dos 40 aos 60 dias, enquanto o H-7974 e Piranão adaptaram-se melhor a curvas do 30 grau.

Os pontos de máximo se situaram entre 101 e 108 dias, e as quantidades máximas calculadas foram semelhantes às apresentadas aos 100 dias. Como estas foram diferentes entre cultivares, pode-se inferir que quantidades máximas também o foram, sendo que o contraste foi entre o cultivar Piranão e os demais. O ponto de inflexão principal (isto é, para a porção maior da curva), para as curvas de 40 grau, e o das curvas de 30 grau, são apresentadas a seguir:

\begin{tabular}{lccc}
\hline Cultivar & $\begin{array}{c}\text { Ponto de máximo } \\
\text { (dias) }\end{array}$ & $\begin{array}{c}\text { Quantidade máxima } \\
\text { (mg/planta) }\end{array}$ & $\begin{array}{c}\text { Ponto de inflexão } \\
\text { (dias) }\end{array}$ \\
\hline \hline Agroceres 256 & 104 & 2,922 & 85 \\
Agroceres 504 & 104 & 3,490 & 76 \\
Centralmex & 108 & 3,193 & 88 \\
H-7974 & 101 & 3,009 & 63 \\
Piranão & 103 & 2,060 & 61 \\
\hline
\end{tabular}

Vale salientar, que a diferença na quantidade de $\mathrm{Cu}$ entre o cultivar Piranão e os outros relacionou-se apenas à parte vegetativa, pois as espigas apresentavam quantidades similares.

Não se pode estabelecer comparações da quantidade de $\mathrm{Cu}$ encontrada com a apresentada por outros autores, havendo muita divergência na literatura. BENNE et al. (1964), citados em OLSON \& LUCAS (1967), encontraram cerca de $4 \mathrm{mg} /$ planta, cerca do dobro do encontrado neste trabalho. Mas, Morrisson (1951), citado em MALAVOLTA et al. (1974), encontrou teores de 4,0 ppm nos grãos e 4,6 ppm no resto da planta, exceto espiga, inferior ao encontrado no presente trabalho.

CLARK \& BROWN (1974a) cultivaram diversas linhagens de milho em solos deficientes em $\mathrm{Cu}$, não verificando sintomas de deficiência em nenhum deles. Aos 21 dias após o plantio, encontraram uma concentração de 6,3 a 12,2 ppm, e citam como niveis deficientes de 4 a 5 ppm. Assim, os cultivares empregados no presente ensaio apresentaram concentrações adequadas de $\mathrm{Cu}$ no início do ciclo vegetativo.

\section{Ferro}

Os dados analíticos relativos à concentração e quantidade de Fe nos órgãos dos cultivares encontram-se no Quadro 2. 


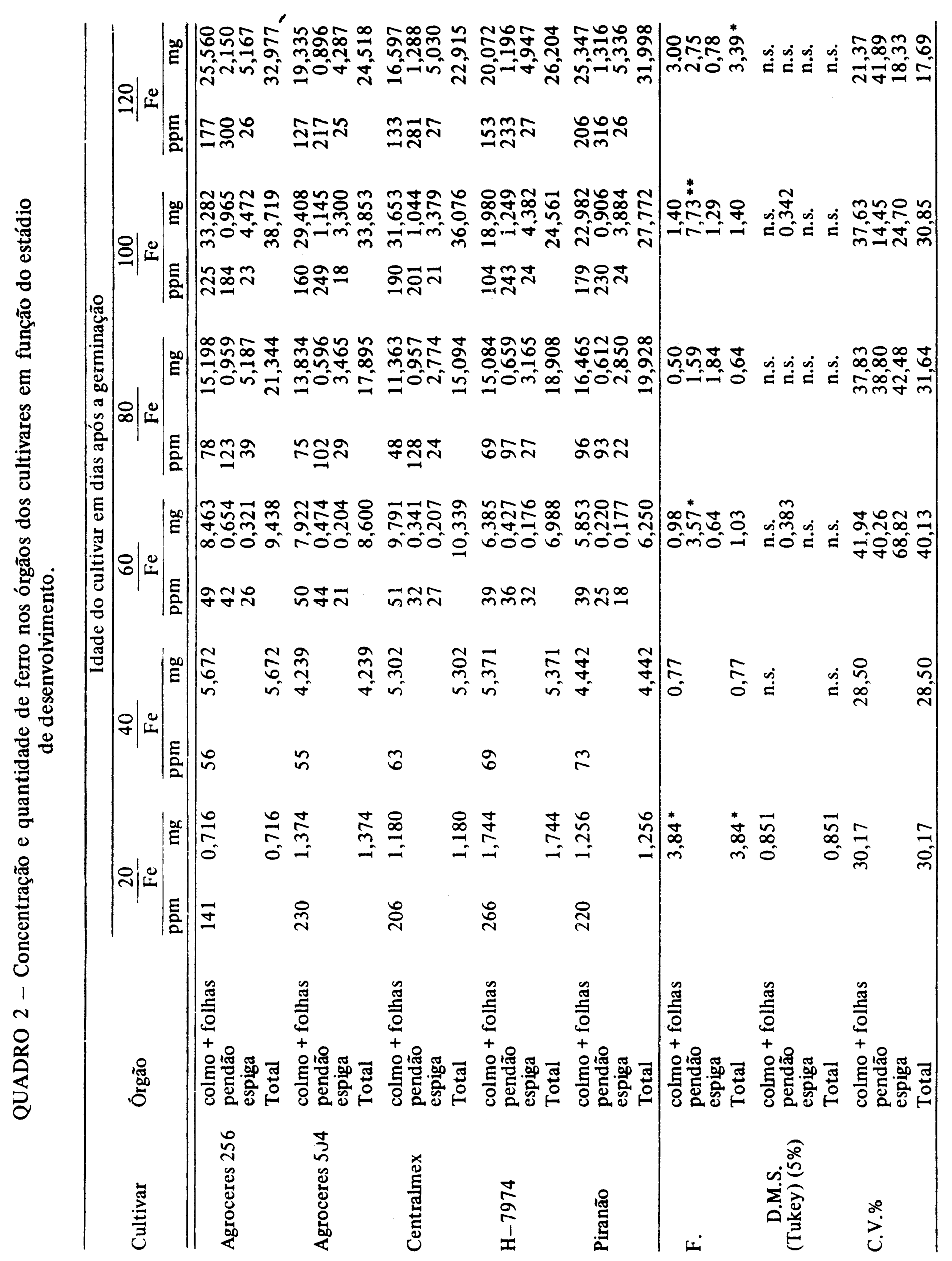


Pelos valores de $\mathrm{F}$ encontrados, verificou-se que, para parte vegetativa só houve diferenças entre cultivares aos 20 dias, para os pendões aos 60 e 100 dias, e para as espigas aos 120 dias.

Os cuitivares diferiram quanto à época de maior acumulação relativa, e assim as curvas de regressão foram diferentes quanto ao grau. Os cultivares Agroceres 256, H-7974 e Piranão seguiram curvas do 30 grau, enquanto o Agroceres 504 e Centralmex seguiram curvas do 40 grau. A representação gráfica das curvas acha-se na Figura 2. A quantidade máxima extraída, e a época em que, teoricamente, a planta possuia esta quantidade são apresentados a seguir:

\begin{tabular}{lccc}
\hline Cultivar & $\begin{array}{c}\text { Ponto de máximo } \\
\text { (dias) }\end{array}$ & $\begin{array}{c}\text { Quantidade máxima } \\
\text { (mg/planta) }\end{array}$ & $\begin{array}{c}\text { Ponto de inflexão } \\
\text { (dias) }\end{array}$ \\
\hline \hline Agroceres 256 & 111 & 36,289 & - \\
Agroceres 504 & 106 & 34,678 & - \\
Centralmex & 106 & 35,982 & - \\
H-7974 & 116 & 26,665 & 71 \\
Piranão & 120 & 32,206 & 76 \\
\hline
\end{tabular}

Mesmo apresentando maior concentração de $\mathrm{Fe}$ no início do ciclo, o cultivar H-7974 apresentou dos 60 dias e aos 100, as menores concentraçбes, enquanto o cultivar Agroceres 256, que apresentou a menor concentração dos 20 dias, teve as maiores no periodo de 60 a 120 dias. Um dos fatores determinantes desse comportamento deve ser a interação com P, Mg e Mn (OLSEN, 1972; WATANABE, 1969; BROWN \& BELL, 1969; BROWN et al., 1972; LUTZ et al., 1972).

A possibilidade de contaminação e as interferências na sua análise, alẻm da variação dos teores nas plantas (JONES, 1972), devem ter acarretado um coeficiente de variação alto, que encobriu a variação existente entre os cultivares.

Comparando-se os dados obtidos com os apresentados por BROWN \& BELL (1969); LUTZ et al. (1972) e CLARK \& BROWN (1974a), verificou-se que a quantidade extraída pelos cultivares utilizados foi superior à apresentada por estes pesquisadores, estando dentro dos limites citados por JONES (1972) e GORSLINE et al. (1965).

Segundo BROWN (1967) e BROWN \& BELL (1969), quando as plantas crescem em solo com teor adequado de $\mathrm{Fe}$ as diferenças entre cultivares, em relação à extração deste nutriente, tende a desaparecer. É provável que este fato tenha contribuido para não se detectar diferenças entre os cultivares. 


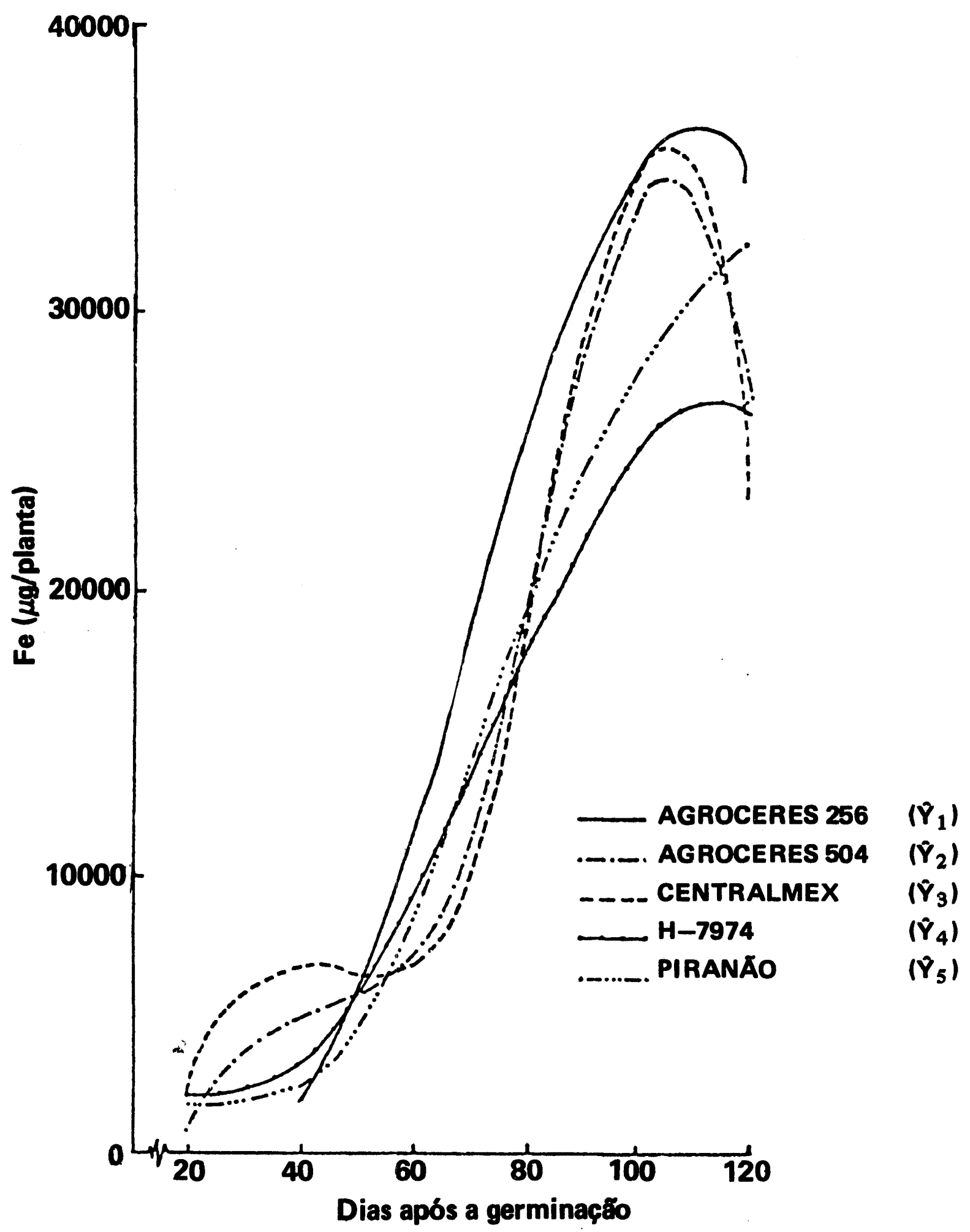

$$
\begin{aligned}
& \hat{Y}_{1}=18.525,81-1.343,91 X+28,645 X^{2}-0,136 x^{3} \quad\left(r^{2}=0,76\right) \\
& \hat{Y}_{2}=-31.751,04+3.075,78 X-92,259 X^{2}+1,140 x^{3}-0,0046 X^{4} \quad\left(r^{2}=0,93\right) \\
& \hat{Y}_{3}=-53.561,61+4.937,09 X-141,20 X^{2}+1,646 x^{3}-0,0064 X^{4} \quad\left(r^{2}=0,86\right) \\
& \hat{Y}_{4}=9.756,48-641,41 X+14,79 X^{2}-0,069 x^{3} \quad\left(r^{2}=0,87\right) \\
& \hat{Y}_{5}=11.436,85-788,36 X+16,94 x^{2}-0,0744 X^{3} \quad\left(r^{2}=0,93\right)
\end{aligned}
$$

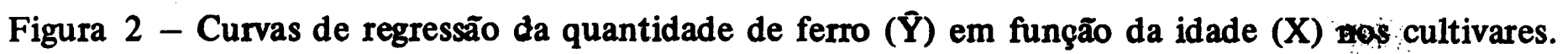




\section{Manganês}

Os dados analíticos referentes à concentração e quantidade de manganês nos órgãos dos cultivares encontram-se no Quadro 3.

Pelos dados expostos pode-se verificar que o comportamento dos cultivares foi semelhante ao relativo ao $\mathrm{Cu}$ e $\mathrm{Fe}$. Diferenças significativas só foram encontradas para a parte vegetativa aos 120 dias, para os pendões aos 60 e 120 dias e para as espigas aos 100 dias.

A diferença entre individuos foi acentuada e superou as diferenças entre cultivares. Aos 60 dias a quantidade de Mn no cultivar Centralmex foi mais que o dobro da contida nas plantas do cultivar Piranão; mesmo assim, não houve diferença significativa entre estes cultivares.

A evolução da quantidade de Mn nas plantas pode ser explicada por uma equação do 20 grau, embora os coeficientes de determinação dessas curvas tenham sido os mais baixos em relação aos elementos pesquisados. As curvas e equações correspondentes acham-se na Figura 3.

Os pontos de máximo variaram de 82 a 94 dias, sendo os cultivares H-7974 e Piranão os mais tardios.

O Centralmex foi o cultivar que menos acumulou $\mathrm{Mn}$, como se pode ver adiante. Como as curvas são de 2 ? grau, não possuem ponto de inflexão.

\begin{tabular}{lcc}
\hline Cultivar & $\begin{array}{c}\text { Ponto de máximo } \\
\text { (dias) }\end{array}$ & $\begin{array}{c}\text { Quantidade máxima } \\
\text { (mg/planta) }\end{array}$ \\
\hline \hline Agroceres 256 & 82 & 14,390 \\
Agroceres 504 & 83 & 11,485 \\
Centralmex & 83 & 9,922 \\
H-7974 & 94 & 14,343 \\
Piranão & 90 & 11,015 \\
\hline
\end{tabular}

$\mathrm{O}$ Mn é um micronutriente que apresenta uma faixa de teores adequados mais ampla. JONES (1972), cita que de 20 a 200 ppm na folha madura é adequado. Outros fatores como a acidez do solo (FRIED \& PEECH, 1946; MOORE, 1972), e a adubação nitrogenada (OLSON \& LUCAS, 1967), também influem na concentração deste micronutriente.

Benne et al. (1964) citados em OLSON \& LUCAS (1967), encontraram cerca de 10-11 mg/planta na maturação fisiológica, no que concordou o presente trabalho.

O Mn é um dos elementos que pose ser lavado das folhas (TUKEY et al. 1958), acarretando perdas deste nutriente durante o ciclo vegetativo.

Mesmo sabendo que a acumulação de Mn é geneticamente controlada (GORSLINE et al., 1964) é provável que a interação genótipo-ambiente seja um fator tão importante quanto o genótipo em si.

Pelos dados obtidos, pode-se afirmar que o cultivar Centralmex deve, em idênticas condições, absorver menor quantidade de Mn que os outros cultivares testados. 


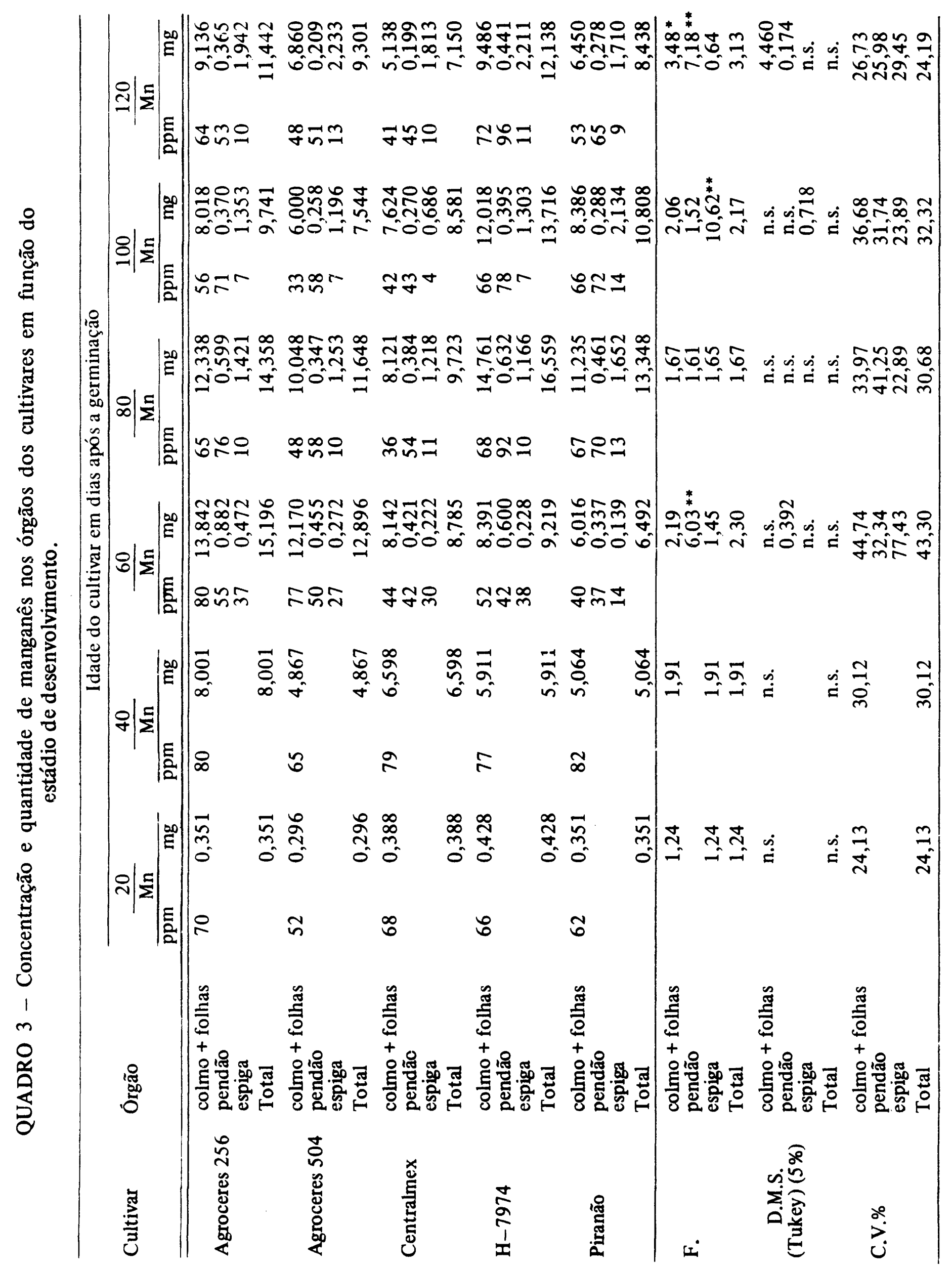




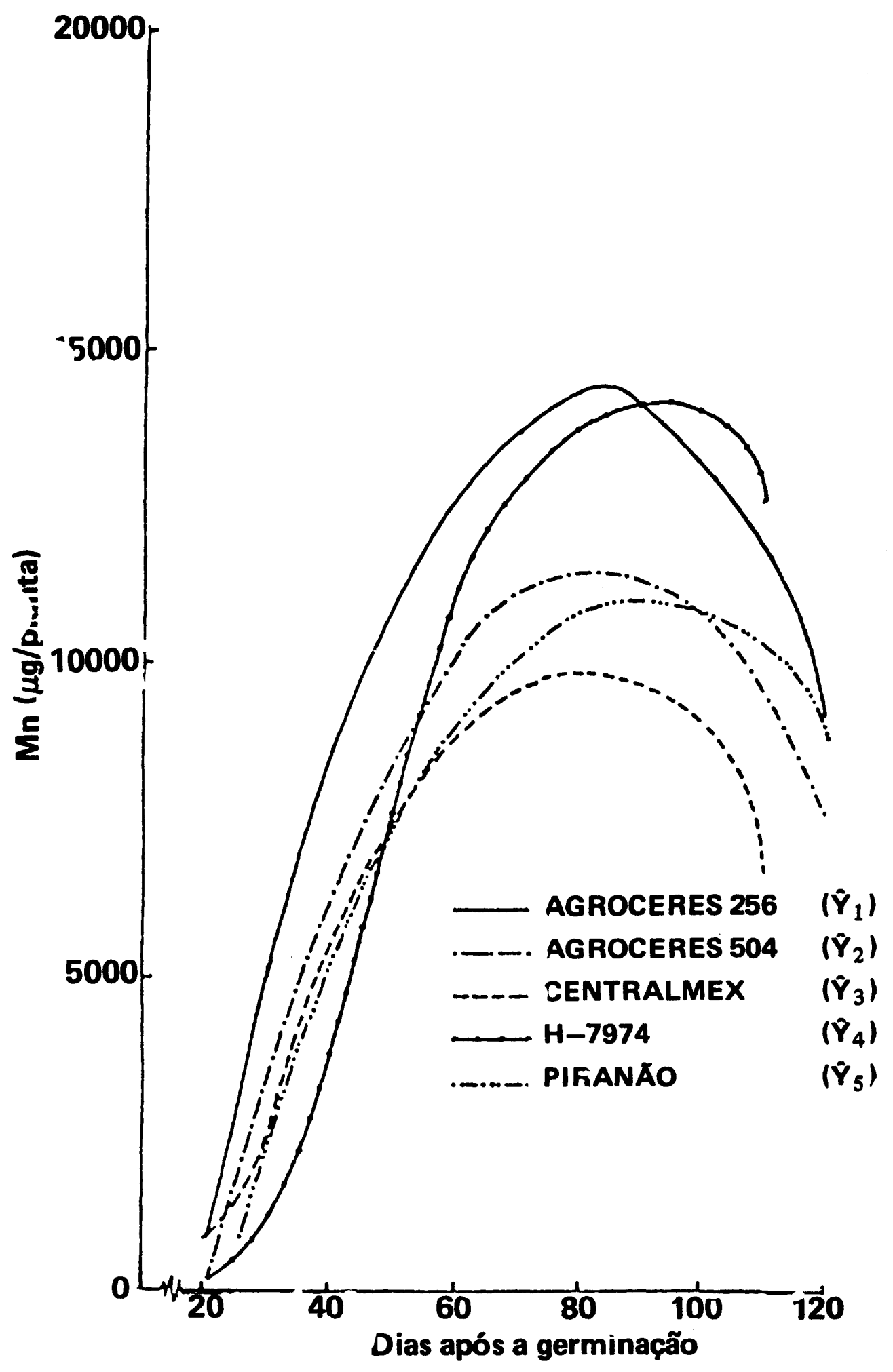

$$
\begin{array}{llll}
\hat{Y}_{1}=-8.967,45+566,68 & X-3,437 X^{2} & \left(r^{2}=0,61\right) \\
\hat{Y}_{2}=-7.827,83+464,296 x-2,7905 X^{2} & \left(r^{2}=0,60\right) \\
\hat{Y}_{3}=-5.785,87+380,15 & X-2,30 & X^{2} & \left(r^{2}=0,53\right) \\
\hat{Y}_{4}=-9.253,66+502,01 & X-2,67 & x^{2} & \left(r^{2}=0,75\right) \\
\hat{Y}_{5}=-7.583,68+412,75 & X-2,29 & x^{2} & \left(r^{2}=0,73\right)
\end{array}
$$

Figura 3 - Curvas de regressão da quantidade de manganês $(\hat{\mathrm{Y}})$ em função da idade $(\mathrm{X})$ nos cultivares. 
Zinco

Os dados analíticos referentes à concentração e quantidade de $\mathrm{Zn}$ nos órgãos dos cultivares encontram-se no Quadro 4.

Pelos valores de $\mathrm{F}$ encontrados, verificou-se que somente aos 20 e 80 dias houve diferenças significativas entre os cultivares, para a parte vegetativa, e aos 60 dias para 0 pendão.

As curvas obtidas pela análise de regressão evidenciaram a semelhança de comportamento dos cultivares. Todos os cultivares seguiram curvas do 20 grau, isto é, a taxa de absorção é, teoricamente decrescente a partir dos 20 dias. 0 coeficiente de determinação das curvas esteve entre 0,71 e 0,73 , mostrando que o erro é relativamente alto. As curvas e equações correspondentes acham-se na Figura 4.

Os cultivares diferiram quanto à época em que atingiram a quantidade máxima de $\mathrm{Zn}$, sendo que o Agroceres 504 e Piranão foram os mais tardios. Os cultivares de porte normal apresentaram quantidade máxima de cerca de 6,50 a $6,70 \mathrm{mg} /$ planta, enquanto o Piranão apresentou cerca de $5,90 \mathrm{mg} /$ planta, o que mostra que sua absorção é similar aos outros.

\begin{tabular}{lcc}
\hline Cultivar & $\begin{array}{c}\text { Ponto de máximo } \\
\text { (dias) }\end{array}$ & $\begin{array}{c}\text { Quantidade máxima } \\
\text { (mg/planta) }\end{array}$ \\
\hline \hline Agroceres 256 & 91 & 6,680 \\
Agroceres 504 & 108 & 6,569 \\
Centralmex & 87 & 6,502 \\
H-7974 & 92 & 6,695 \\
Piranão & 107 & 5,882 \\
\hline
\end{tabular}

O solo utilizado no presente trabalho não responde à aplicação de $\mathrm{Zn}$ (BRASIL SOBRo, 1966), donde se deduz que seu teor deste elemento (20-100 ppm) está dentro dos limites satisfatórios.

A concentração de $\mathrm{Zn}$ na planta é um dado relativo, dependente do $\mathrm{pH}$ do solo (LUTZ et al., 1972) e do teor de $P$ nos tecidos (WARNOCK, 1970). Este último autor encontrou teores adequados de 9 a 15 ppm no colmo e 10 a 18 na folha, aos 56 dias após o plantio. WATANABE et al. (1965), encontraram, nos estágios iniciais, $513 \mu \mathrm{g}$ de $\mathrm{Zn} /$ vaso, correspondente a $17 \mathrm{ppm}$ na planta inteira com um teor de $\mathrm{P}$ de $0,28 \%$, como niveis suficientes para $o$ milho. Os dados obtidos no presente trabalho indicaram que os cultivares empregados absorvem quantidades idênticas às citadas na literatura.

Como era de se esperar, a quantidade de $\mathrm{Zn}$ no cultivar Piranão não está ligada ao porte anão, determinado pelo gene $\mathrm{br}_{2}$, embora a deficiência de $\mathrm{Zn}$ também provoque encurtamento dos entrenós (MALAVOLTA et al., 1974). O caráter anão, determinado geneticamente, está ligado ao bloqueio da sintese de giberelinas, sendo, portanto, independente da concentração de $\mathrm{Zn}$. 


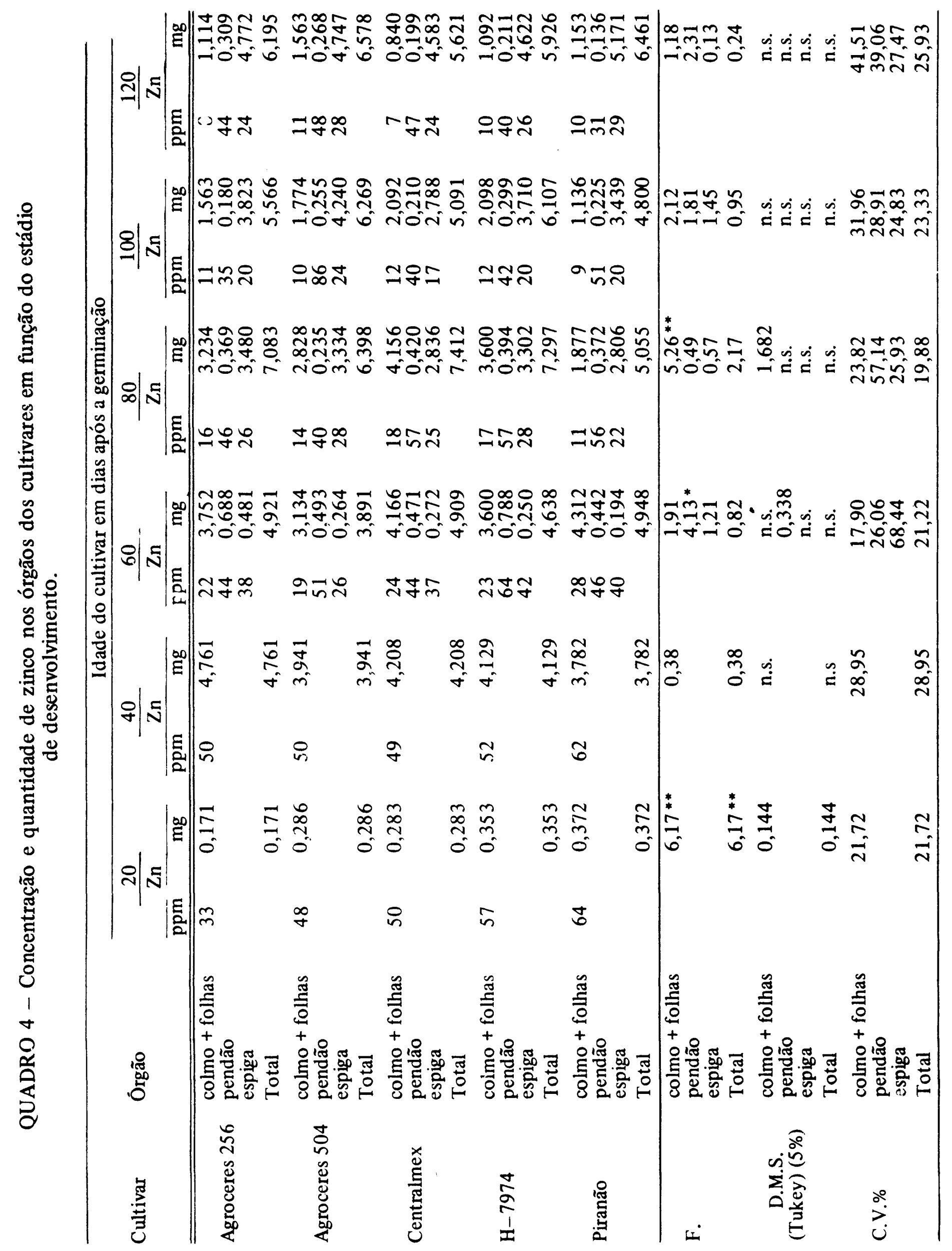




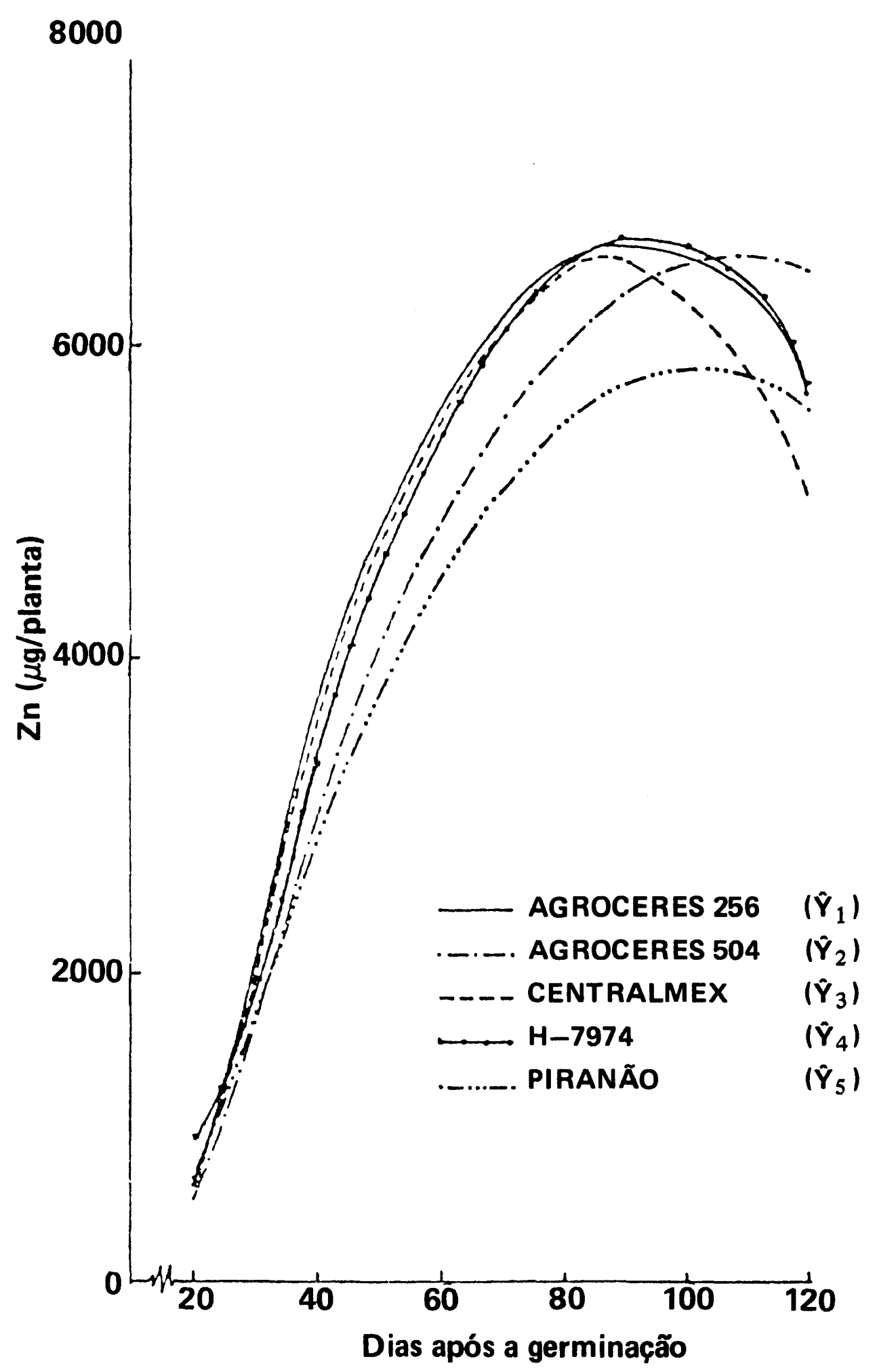

$$
\begin{array}{llll}
\hat{Y}_{1}=-3.105,85+215,28 X-1,184 & X^{2} & \left(r^{2}=0,72\right) \\
\hat{Y}_{2}=-2.374,63+165,00 X-0,761 & X^{2} & \left(r^{2}=0,71\right) \\
\hat{Y}_{3}=-3.440,70+227,13 X-1,297 & X^{2} & \left(r^{2}=0,73\right) \\
\hat{Y}_{4}=-3.334,27+218,22 X-1,187 & X^{2} & \left\langle r^{2}=0,71\right) \\
\hat{Y}_{5}=-1.528,90+138,20 X-0,6443 X^{2} & \left\langle r^{2}=0,71\right)
\end{array}
$$

Figura 4 - Curvas de regressão da quantidade de zinco $(\hat{Y})$ em função da idade $(\mathrm{X})$ nos cultivares. 


\section{Exportação de nutrientes}

Os dados referentes ao teor e quantidade de micronutrientes nas espigas acham-se no Quadro 5.

A análise estatística empreendida mostrou que, em relação à quantidade de nutrientes nos grãos, só houve diferença significativa em relação ao $\mathrm{Mn}$, sendo que o Agroceres 504 apresentou maior quantidade que os outros cultivares.

É interessante assinalar que o cultivar Agroceres 504 (opaco-2) apresentou teores mais elevados em Mn e $\mathrm{Zn}$ que os outros cultivares.

Em relação a "palha + sabugo", encontrou-se diferenças significativas ao nivel de $5 \%$ para o $\mathrm{Cu}$.

Para se avaliar a ordem de grandeza na exportação de nutrientes em uma cultura utilizando estes cultivares, calculou-se a quantidade nas espigas para uma colheita média de $6000 \mathrm{~kg} / \mathrm{ha}$ de grãos, para uma população de 50.000 plantas/ha.

\begin{tabular}{lc}
\hline Nutriente & Quantidade exportada por ha \\
\hline \hline Cobre $(\mathrm{Cu}$ & $26-35 \mathrm{~g}$ \\
Ferro $(\mathrm{Fe})$ & $200-220 \mathrm{~g}$ \\
Manganês $(\mathrm{Mn})$ & $90-140 \mathrm{~g}$ \\
Zinco $(\mathrm{Zn})$ & $160-250 \mathrm{~g})$ \\
\hline
\end{tabular}

Em termos gerais, pode-se afirmar que, mesmo se os cultivares absorverem quantidades diferentes de nutrientes, a sua exportação através das espigas foi similar. 


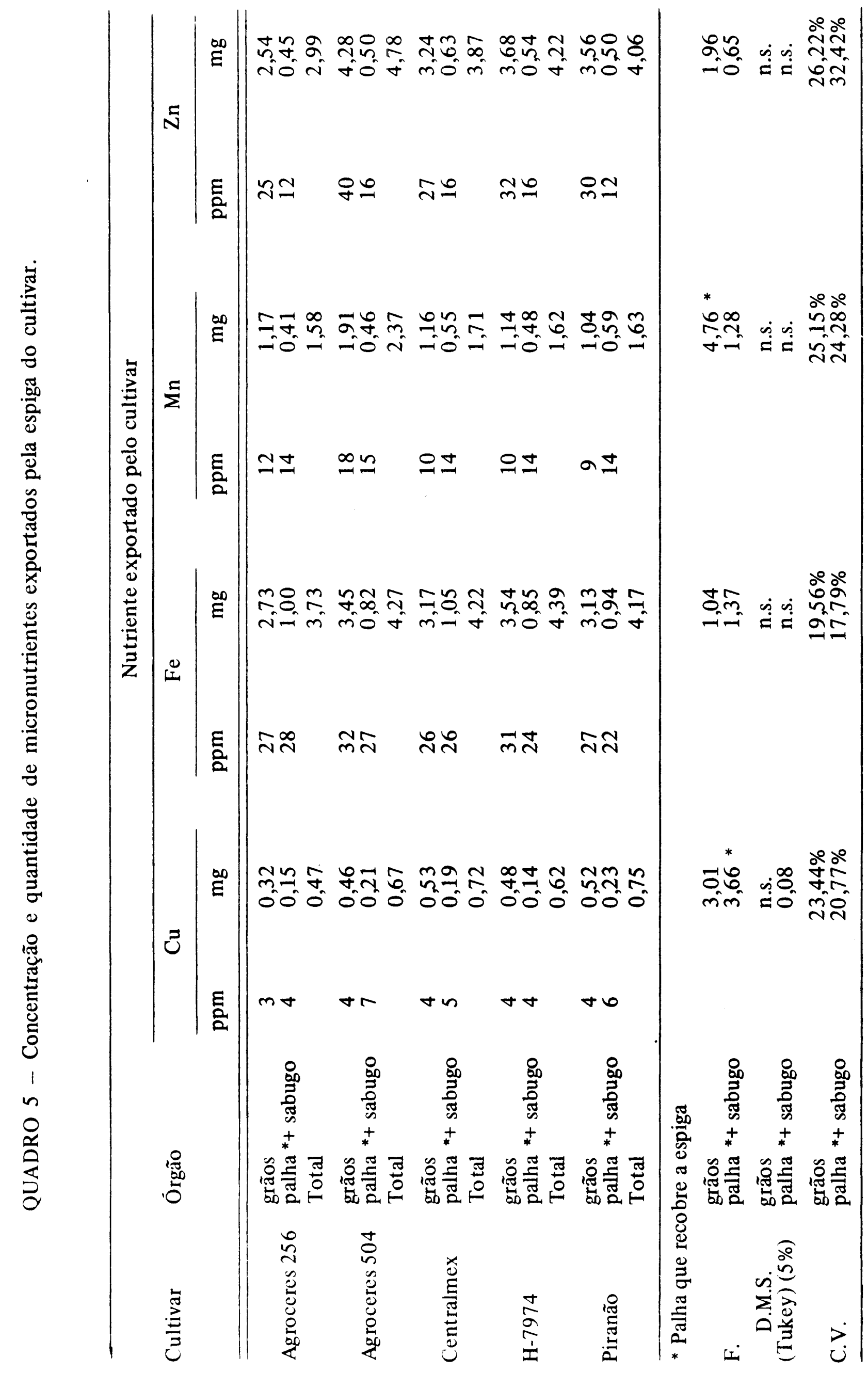




\section{CONCLUSÕES}

\section{Acumulação de nutrientes}

- Diferenças na acumulação de micronutrientes manifestam-se antes da fase de crescimento intenso (20 dias), embora possam não ser mais detectadas posteriormente.

- Há diferenças na taxa de acumulação de alguns nưrientes ( $\mathrm{Zn}$, por ex.) entre cultivares, sem afetar a quantidade máxima acumulada.

- A seleção voltada para a obtenção de plantas, simplesmente mais produtivas deve, salvo casos específicos, levar a plantas com exigências nutricionais semelhantes.

- Os cultivares atingem o máximo da quantidade de nutrientes nas seguintes épocas, em dias: $\mathrm{Cu}$ (101-104); $\mathrm{Fe}$ (106-120); $\mathrm{Mn}$ (82-94); $\mathrm{Zn}(87-108)$.

- As quantidades máximas extraídas pelos cultivares estão dentro dos limites, em mg/planta: $\mathrm{Cu}(2,06-3,49)$; Fe $(26,66-36,29)$; Mn $(9,92-14,39)$ e $\mathrm{Zn}(5,88-6,69)$.

\section{Exportação de nutrientes}

- Não há diferenças na quantidade de nutrientes exportada nos grãos dos cultivares, exceto para o $\mathrm{Mn}$.

- Além da diferença na quantidade de nutrientes translocada para a espiga, pode haver diferenças na distribuição dentro da espiga.

- A exportação de nutrientes nas espigas dos cultivares são da seguinte ordem, por hectare colhido: $\mathrm{Cu}$ (26-35 g); Fe (200-220 g); Mn (90-140 g) e Zn (160-250 g).

\section{SUMMARY}

\section{DIFFERENTIAL ACCUMULATION OF NUTRIENTS BY FIVE CULTIVARS OF CORN (Zea mays L.). II - MICRONUTRIENTS ACCUMULATION}

The objective of the present work was to examine the differences in growth, yield, accumulation and transport of nutrients between the cultivars Agroceres 256, Agroceres 504, Centralmex, H-7974 and Piranão. The experiment was carried out in the municipality of Piracicaba, State of São Paulo, Brazil. The soil type was a sandy oxisol of medium fertility, except for $\mathrm{K}$ which was low. The experimental set-up was a randon block design with four replications. Common cultivation practices were followed, and the fertilizer used consisted of $83 \mathrm{~g}$ of a formula: 30-120-70, per meter lenght at planting, and $33 \mathrm{~g}$ of the formula: 50-0-45 per meter lenght as dressing 22 days after germination. The plant population density was 50,000 per hectare. Plants were collected for analysis at 20 days after germination, and there after at intervals of 20 days up to 120 days. The plants were divided into "stems + leaves", tassels, and ears for chemical analysis of $\mathrm{Cu}, \mathrm{Fe}, \mathrm{Mn}$ and $\mathrm{Zn}$. 


\section{Conclusions:}

\section{Accumulation of nutrients}

- Differences in the accumulation of micronutrients appeared before the period of rapid growth (20 days) although afterwards no differences were detectable.

- The maximum level of nutrients are attained in the following period (in days): $\mathrm{Cu}$ (101-104); Fe (106-120); Mn (82-94); Zn (87-108).

- The maximum quantities taken-up (in mg/plant) are: $\mathrm{Cu}(2.06-3.49)$; $\mathrm{Fe}$ (26.66-36.29); Mn (9.92-14.39); Zn (5.88-6.69).

\section{Transport of nutrients}

- The transport of nutrients into the ears of the different cultivars are (per ha harvested): $\mathrm{Cu}(26-35 \mathrm{~g}) ; \mathrm{Fe}(200-220 \mathrm{~g}) ; \mathrm{Mn}(90-140 \mathrm{~g}) ; \mathrm{Zn}(160-250 \mathrm{~g})$.

\section{LITERATURA CITADA}

BRASIL SOBR 9, M.O.C., 1966. Levantamento do teor de zinco em alguns solos do município de Piracicaba. Piracicaba, SP., 96 pp. Tese (cátedra). ESALQ.

BROWN, J.C., 1967. Differential uptake of Fe and Ca by two corn genotypes. Soil Sci., 193: 331-338.

BROWN, J.C. \& BELL, W.D., 1969. Iron uptake dependent upon genotypes of corn. Soil Sci. Soc. Amer. Proc., 33 99-101.

BROWN, J.C. \& AMBLER, J.E., 1970. Further characterization of iron uptake in two genotypes of corn. Soil Sci. Soc. Amer. Proc., $34: 249-252$.

BROWN, J.C., AMBLER, J.E., CHANEY, R.L. \& FOY, C.E., 1972. Differential response of plant genotypes to micronutrients. IN: Mortvedt, J.J. et al. (eds.). Micronutrients in agriculture. Soil Science Society of America, Inc., Madison, Wis., p. 389-413.

BROWN, J.C. \& CLARK, R.B., 1974. Differential response of two maize imbreds to molybdenum stress. Soil Sci. Amer. Proc., 38:331-333.

CLARK, R.B. \& BROWN, J.C., 1974a. Differential mineral uptake by maize imbreds. Comm. Soil Sci. Plant Anal., 5 :213-227.

DONALD, C.M., 1968. The breeding of crop ideotypes. Euphytica, 17:385-403.

DUNCAN. W.G., 1967. Corn yield to meet the challenge. IN : Maximum crop yield - the challenge. American Society of Agronomy (publicação especial no 9), Madison, Wis., p. 51-56.

EPSTEIN, E., 1972. Mineral nutrition of plants: principles and perspectives. - John Wiley and Sons. Inc., New York, London. $412 \mathrm{p}$.

FRIED, M. \& PEECH, M., 1946. The comparative effects of lime and gypsum upon plants grown on acid soils. J. Amer. Soc. Agron., 38:614-623.

GORSLINE, G.W., THOMAS, W.I. \& BAKER, D.E., 1964. Inheritance of P, K, Mg, Cu, B, Zn, Mn, $\mathrm{Al}, \mathrm{Fe}$ concentration by com (Zea mays L.) leaves and grain. Crop Sci., 4:207-210.

GORSLINE, G.M., BAKER, D.E. \& THOMAS, W.I., 1965. Accumulation of eleven elements by field corn (Zea mays L.). The Pennsylvania Agricultural Experiment Station. Bull. 725. 
GREGORY, F.G. \& CROWTHER, F., 1928. A physiological study of varietal difference in plants. Part I: A study of the comparative yields of barley varieties with different manurings. Ann. Bot., 42:757-770.

JONES, J.B., Jr., 1972. Plant tissue analysis for micronutrients. IN: Mortvedt, J.J. et al. (eds.). Micronutrients in agriculture. Soil Science Society of America, Inc., Madison, Wis., p. 319-346.

LOCKMAN, R.B., 1969. Relationships between corn yields and nutrient concentration in seedling whole-plant samples. Agron. Abstr., p. 97. American Society of Agronomy, Madison, Wis.

LUTZ, J.A., Jr., GENTER, C.F. \& HAWKINS, G.W., 1972. Effect of soil pH on element concentration and uptake by maize. I:P, K, Ca, Mg and Na. Agron., J., 64 :581-583.

MALAVOLTA, E., HAAG, H.P., MELLO, F.A.F. \& BRASIL SOBRO, M.O.C., 1974. Nutrição mineral e adubação de plantas cultivadas. Liv. Pioneira editora, São Paulo, 727 pp.

MOORE, D.P., 1972. Mechanisms of micronutrients uptake by plants. IN: Mortvedt, J.J. et al. (eds.). Micronutrients in agriculture. Soil Science Society of America, Inc. Madison, Wis., p. 171-198.

NEUBERT, P., WRAZIDLO, W., VIELEMEYER, N.P., HUNDT, I., GULMICK, F. \& BERGMANN, W., 1969. Tabellon zur planzenanalyzeerste orietierende ubersicht. Institut fur planzenernahrung. Jena, Berlin.

OLSEN, S.R., 1972. Micronutrients interactions. IN : Mortvedt, J.J. et al. (eds.). Micronutrients in agriculture. Soil Science of America, Inc. Madison, Wis., p. 243-264.

OLSON, R.A. \& LUCAS, R.E., 1967. Fertility requirement: secundary and micronutrients. IN: Pierre, W.H. et al. (eds.). Advances in corn production: principles and practices. The lowa State University Press. Ames, Iowa., p. 285-330.

RANZANI, G., 1956. Levantamento da carta de solo da Seç̧ão Técnica "Química Agrícola" da Escola Superior de Agricultura "Luiz de Queiroz". Piracicaba, SP. Tese (livre-docente) ESALQ.

SARRUGE, J.R. \& HAAG, H.P., 1974. Análises químicas em plantas. Piracicaba, SP. Departamento de Química, ESALQ (publicação especial).

SPRAGUE, G.F., 1969. Germ Plasm manipulation in the future. IN: J.D. Eastin et al. (eds.). Physiological aspects of crop yield. American Society of Agronomy. Crop Sci. Society of America, Madison, Wis., p. 375-395.

TUKEY, H.B., TUKEY JR., H.B. \& WITTWER, S.H., 1958. Loss of nutrients by foliar leaching as determined by radioisotopes. Proc. Amer. Soc. Hort. Sci., 71 :496-506.

WARNOCK, R.E., 1970. Micronutrient uptake and mobility within corn plants (Zea mays L.) in relation to phosphorus - induced zinc deficiency. Soil Sci. Soc. Amer. Proc., 34:765-769.

WATANABE, F.S., LINDSAY, W.L. \& OLSEN, S.R., 1965. Nutrient balance involving phosphorus, iron, and zinc. Soil Sci. Amer., 29:562-565. 
